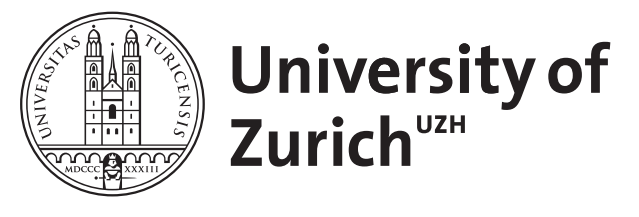

\title{
Partition function of periodic isoradial dimer models
}

\author{
de Tilière, B
}

\begin{abstract}
Isoradial dimer models were introduced in Kenyon (Invent Math 150(2):409-439, 2002) they consist of dimer models whose underlying graph satisfies a simple geometric condition, and whose weight function is chosen accordingly. In this paper, we prove a conjecture of (Kenyon in Invent Math 150(2):409-439, 2002), namely that for periodic isoradial dimer models, the growth rate of the toroidal partition function has a simple explicit formula involving the local geometry of the graph only. This is a surprising feature of periodic isoradial dimer models, which does not hold in the general periodic dimer case (Kenyon et al. in Ann Math, 2006).
\end{abstract}

DOI: https://doi.org/10.1007/s00440-006-0041-2

Posted at the Zurich Open Repository and Archive, University of Zurich ZORA URL: https://doi.org/10.5167/uzh-21580

Journal Article

Accepted Version

Originally published at:

de Tilière, B (2007). Partition function of periodic isoradial dimer models. Probability Theory and Related Fields, 138(3-4):451-462.

DOI: https://doi.org/10.1007/s00440-006-0041-2 


\title{
Partition function of periodic isoradial dimer models
}

\author{
Béatrice de Tilière * \\ Institut für Mathematik, Universität Zürich, Winterthurerstrasse 190, CH-8057 Zürich. \\ beatrice.detiliere@math.unizh.ch
}

\begin{abstract}
Isoradial dimer models were introduced in [12] - they consist of dimer models whose underlying graph satisfies a simple geometric condition, and whose weight function is chosen accordingly. In this paper, we prove a conjecture of [12], namely that for periodic isoradial dimer models, the growth rate of the toroidal partition function has a simple explicit formula involving the local geometry of the graph only. This is a surprising feature of periodic isoradial dimer models, which does not hold in the general periodic dimer case [14].
\end{abstract}

\section{Introduction}

In this paper, we solve a conjecture of [12], namely that the growth rate of the partition function of periodic isoradial dimer models can be expressed explicitly, using only the local geometry of the underlying graph. This is a surprising feature of isoradial dimer models, which does not hold in the case of general periodic dimer models. Indeed, in the general periodic case, Kenyon, Okounkov and Sheffield [14] obtain an expression for the growth rate of the partition function involving elliptic integrals, thus making it hard to do explicit computations. An example of application of our result yields a two line proof of Kasteleyn's celebrated result for the growth rate of the dimer partition function of the quadratic lattice $\mathbb{Z}^{2}[7,8]$, see Section 1.5. In order to state our result, let us precisely describe the setting.

\subsection{Dimer model}

The dimer model belongs to the field of statistical mechanics, and represents the adsorption of diatomic molecules on the surface of a crystal, it is defined in the following way. The surface of the crystal is modeled by a graph $G$. We assume that $G$ is simple, infinite, simply connected (i.e. it is the one skeleton of a simply connected union of faces) and that its vertices are of degree $\geq 3$. A dimer configuration of $G$ is a perfect matching of $G$, that is a subset of edges $M$, such that every vertex of $G$ touches a unique edge of $M$; refer to Figure 1 for an example in the case where $G$ is a finite subgraph of $\mathbb{Z}^{2}$. Let us denote by $\mathcal{M}(G)$ the set of perfect matchings of $G$.

\footnotetext{
* Supported by Swiss National Fund under grant 47102009
} 


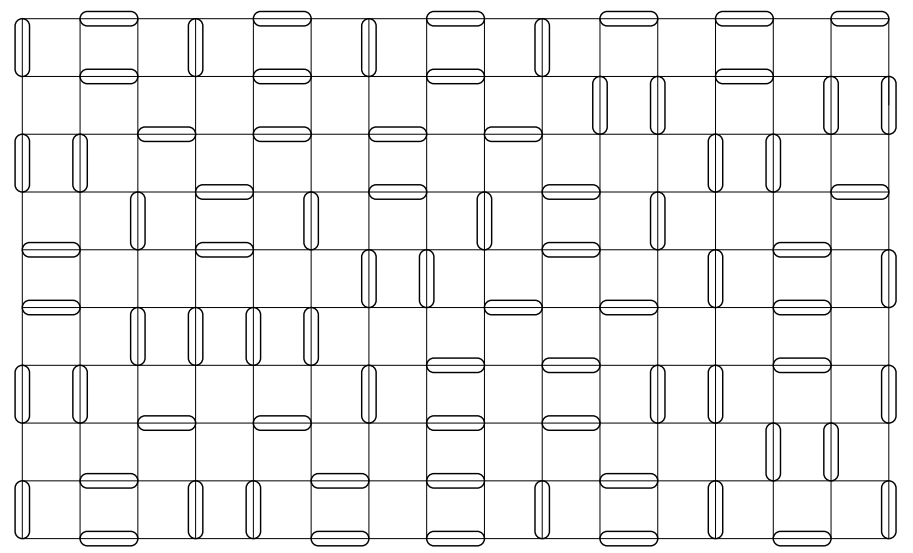

Figure 1: A dimer configuration of a finite subgraph of $\mathbb{Z}^{2}$.

Consider a simply connected finite subgraph $G_{0}$ of $G$. Then, dimer configurations of $G_{0}$ are chosen with respect to the Boltzmann measure, defined in the following way. Assume that a positive weight function $\nu$ is assigned to edges of $G$, that is, each edge $e$ of $G$ has a weight $\nu(e)$. Then, each dimer configuration $M$ of $G_{0}$ has an energy $\mathcal{E}(M)=-\sum_{e \in M} \log \nu(e)$. The probability of occurrence of the dimer configuration $M$ chosen with respect to the Boltzmann measure $\mu_{0}$, is given by:

$$
\mu_{0}(M)=\frac{e^{-\mathcal{E}(M)}}{Z\left(G_{0}, \nu\right)}=\frac{\prod_{e \in M} \nu(e)}{Z\left(G_{0}, \nu\right)}
$$

where $Z\left(G_{0}, \nu\right)=\sum_{M \in \mathcal{M}\left(G_{0}\right)} \prod_{e \in M} \nu(e)$ is the normalizing constant, known as the partition function.

The first step in the study of the dimer model is the computation of the partition function. Indeed, the latter yields precious information about the global behavior of the system $[1,2,14]$, whose understanding is the goal of statistical mechanics. Kasteleyn $[7,8]$, and independently Temperley \& Fisher [20], laid the ground stone for the study of the partition function. Consider $G_{0}$ as above. Then they give an explicit formula for $Z\left(G_{0}, \nu\right)$ as the square root of the determinant of a matrix $\mathrm{K}_{0}$, also known as a Kasteleyn matrix. Loosely stated, $\mathrm{K}_{0}$ is the weighted adjacency matrix of $G_{0}$ (weighted by the function $\nu$ ), where minus signs are added to coefficients in a suitable way, see Section 2.2 for details.

Since edges of dimer configurations represent diatomic molecules, the next step is to understand the partition function of infinite graphs. The relevant question then, is the computation of its growth rate. More precisely, if $\left\{G_{n}\right\}$ is an exhaustion of $G$ by finite 
graphs, the goal is to compute:

$$
C=\lim _{n \rightarrow \infty} \frac{1}{n^{2}} \log Z\left(G_{n}, \nu\right)
$$

Note that this limit is not universal, in the sense that it strongly depends on the choice of exhaustion $\left\{G_{n}\right\}$. Moreover, in the case of exhaustions by planar graphs, this limit is hard to compute because of the lack of symmetry which reflects in the Kasteleyn matrix. This is the reason why computations are done on toroidal graphs, as explained in the next section.

\subsection{Toroidal dimer models}

Consider an infinite graph $G$ as above, and suppose that $G$ is bipartite, i.e that it admits a bipartite coloring of its vertices. Assume moreover that there exists a bi-dimensional lattice $\Lambda$, such that $G$ is doubly $\Lambda$-periodic, that is the graph $G$ as well as its vertex coloring are bipartite. Define $G_{n}$ to be the toroidal graph $G / \Lambda n$, then $\left\{G_{n}\right\}$ is a natural exhaustion of the graph $G$.

In the case where $G$ is the square lattice $\mathbb{Z}^{2}$, Kasteleyn $[7,8]$ gives an explicit expression for $Z\left(G_{n}, \nu\right)$ as a linear combination of the determinants of four Kasteleyn matrices. Tesler [21] generalizes this result to any graph $G$ satisfying the above, see Section 2.2 for details (he actually generalizes it to graphs embedded on genus $g$ surfaces). In [14], Kenyon, Okounkov and Sheffield give an explicit expression for the limit $C$ of equation (1) in the case of the natural toroidal exhaustion $\left\{G_{n}\right\}$ of $G$. For general periodic, bipartite graphs $G$, this limit depends on the combinatorics of the model and involves elliptic integrals.

In the next section, we define isoradial dimer models - the sub-family of dimer models for which the expression for the growth rate $C$ of (1) becomes surprisingly simple.

\subsection{Isoradial dimer models}

Isoradial dimer models were introduced in [12]. They are dimer models on graphs $G$ satisfying a geometric condition called isoradiality. This notion first appeared in [5], see also [16], and is defined as follows. All faces of $G$ are inscribable in a circle, and all circumcircles have the same radius, moreover, all circumcenters of the faces are contained in the closure of the faces. The common radius is taken to be 1. An isoradial embedding of the dual graph $G^{*}$ is obtained by sending dual vertices to the center of the corresponding faces.

Recall that the energy of a dimer configuration depends on the weights assigned to edges of $G$. In the case of isoradial graphs, one considers a specific weight function $\nu$, called the critical weight function, defined as follows [12]. To each edge $e$ of $G$ corresponds a unit side-length rhombus $R(e)$ whose vertices are the vertices of $e$ and of its dual edge $\left(R(e)\right.$ may be degenerate). Let $\widetilde{R}=\cup_{e \in G} R(e)$. Then, define $\nu(e)=2 \sin \theta$, 
where $2 \theta$ is the angle of the rhombus $R(e)$ at the vertex it has in common with $e ; \theta$ is called the rhombus angle of the edge $e$. Note that $\nu(e)$ is the length of $e^{*}$ the dual edge of $e$.

When the graph considered for a dimer model is isoradial, and when the energy of configurations is determined by the critical weight function, we speak of an isoradial dimer model.

\subsection{Result}

Consider an isoradial dimer model. Assume moreover that $G$ is bipartite and doubly $\Lambda$-periodic. As above consider the natural exhaustion $\left\{G_{n}\right\}$ of $G$ by toroidal graphs. Then, Theorem 1 below gives an explicit expression for the growth rate of the partition function of the exhaustion $\left\{G_{n}\right\}$ as a function of the local geometry of the graph.

\section{Theorem 1}

$$
\lim _{n \rightarrow \infty} \frac{1}{n^{2}} \log Z\left(G_{n}, \nu\right)=\sum_{i=1}^{m}\left(\frac{\theta_{i}}{\pi} \log 2 \sin \theta_{i}+\frac{1}{\pi} L\left(\theta_{i}\right)\right),
$$

where, $\theta_{1}, \ldots, \theta_{m}$ are the rhombus angles of the edges $e_{1}, \ldots, e_{m}$ of $G_{1}$, and $L$ is Lobachevsky's function, $L(x)=-\int_{0}^{x} \log 2 \sin t d t$.

- The right hand side of (2) is (up to a factor 2), the expression Kenyon obtained for what he calls the log of the normalized determinant of the Dirac operator [12]. He conjectured it to be the right limit for the growth rate of the partition function of an appropriate exhaustion of $G$. Theorem 1 states that this is the right limit in the case of the natural exhaustion $\left\{G_{n}\right\}$ of $G$ by toroidal graphs.

- The surprising feature of Theorem 1 is that the growth rate of the partition function is expressed using geometric information of the fundamental domain $G_{1}$ only, no combinatorics is involved. This is contrast with the result of [14] for general bipartite periodic graphs. Note that, although the result of [14] is true in a more general setting, there seems to be no simple way of re-deriving Theorem 1 from it. Let us also mention that we use the result of [14] in order to have a priori existence of the limit $C$ of equation (1).

\subsection{Example: Kasteleyn's computation}

In $[7,8]$, Kasteleyn skillfully computes the toroidal partition function for $G=\mathbb{Z}^{2}$. As an example of application of Theorem 1, let us rederive his result in a two line calculation. Note that the following computation was already mentioned in [12], as a support for the conjecture proved in Theorem 1 . When $G=\mathbb{Z}^{2}$, the critical weight function is $\nu \equiv \sqrt{2}$, see Figure 2 (left). Moreover, consider the fundamental domain $G_{1}=G / \Lambda$ as in Figure 2 (right). 

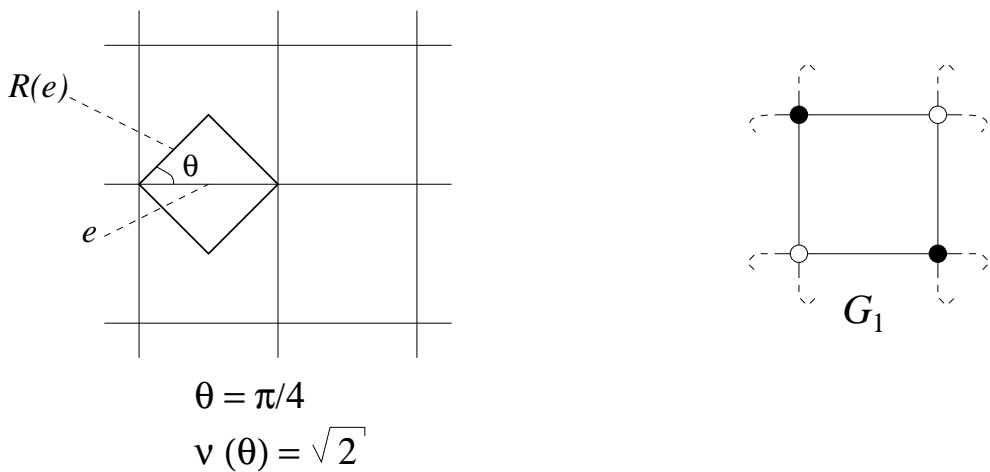

Figure 2: Critical weight function for $\mathbb{Z}^{2}$ (left). Fundamental domain $G_{1}$ (right).

Then, a direct computation using Theorem 1 yields:

$$
\begin{aligned}
\lim _{n \rightarrow \infty} \frac{1}{2 n^{2}} \log Z\left(G_{n}, \sqrt{2}\right) & =4\left(\frac{1}{4} \log \sqrt{2}+\frac{1}{\pi} L\left(\frac{\pi}{4}\right)\right), \\
& =\frac{1}{2} \log 2+\frac{2 \mathcal{K}}{\pi}
\end{aligned}
$$

where $\mathcal{K}$ is Catalan's constant. Note that the $\frac{1}{2} \log 2$ factor differs from Kasteleyn's computation, because we have weights $\sqrt{2}$ on the edges instead of weights 1 .

Acknowledgments: we would like to thank Richard Kenyon for proposing the question solved in this paper, and for fruitful discussions.

\section{Proof of Theorem 1}

In the whole of this section, we let $G$ be an infinite, bipartite, $\Lambda$-periodic, isoradial graph. We assume that edges of $G$ are assigned the critical weight function. Moreover, $W$ denotes the set of white vertices of $G, B$ the set of black ones, and $G_{n}$ the toroidal graph $G / \Lambda n$.

Before giving the proof of Theorem 1, let us precisely describe the conjecture of [12]. In [12], Kenyon introduces the Dirac operator $K$ indexed by vertices of $G$ (we refer the reader to [12] for the definition of $K$, and to Section 2.1 for the definition of the real Dirac operator $\mathrm{K}$ which is closely related). He also defines its inverse $K^{-1}$, for which he proves existence and uniqueness. One of his beautiful result is an explicit expression for the log of the normalized determinant "log $\operatorname{det}_{1} K$ " of the Dirac operator $K$, defined by the following PDE:

$$
\frac{\partial \log \operatorname{det}_{1} K}{\partial K(w, b)}=\frac{1}{\left|V\left(G_{1}\right)\right|} K^{-1}(b, w)
$$


where $w \in W, b \in B$ are adjacent vertices, $\left|V\left(G_{1}\right)\right|$ is the number of vertices of $G_{1}$. Adding some initial condition allows him to solve the PDE, and obtain the explicit expression for $\log \operatorname{det}_{1} K$, which is the right hand side of equation (2). How is this related to the dimer model? The Dirac operator $K$ is closely related to an infinite Kasteleyn matrix - to have an actual infinite Kasteleyn matrix, one needs to work with the real Dirac operator K (see Section 2.1 below), obtained from the Dirac operator by a gauge transformation. If $G_{0}$ is a finite subgraph of $G$, and $\mathrm{K}_{0}$ the restriction of $\mathrm{K}$ to the vertices of $G_{0}$, then $\log \operatorname{det}_{1} \mathrm{~K}_{0}$ can be defined naturally as $\frac{1}{V\left(G_{0}\right) \mid} \log \operatorname{det} \mathrm{K}_{0}$, which is the normalized log of the dimer partition function of $G_{0}$. Hence, the conjecture is to interpret (3) as the limit of the dimer partition function on some appropriate exhaustion of $G$. The problem lies in proving the existence of such limits, leading us to work with toroidal exhaustions. Note that part of the proof is close to [12], since it consists in solving the PDE (3) for operators restricted to subgraphs of the exhaustion.

The structure of Section 2 is as follows. Section 2.1 consists in the definition of the real Dirac operator $\mathrm{K}$ and its inverse $\mathrm{K}^{-1}$. The operator $\mathrm{K}$ was introduced in [3], see also [13]. Note that features of $K$ are closely related to those of the Dirac operator $K$ of [12]. In Section 2.2, we state the explicit expression of $[8,21]$ for the toroidal partition function. Section 2.3 uses Sections 2.1 and 2.2, and consists in the proof of Theorem 1.

\section{$2.1 \quad$ Real Dirac operator}

The real Dirac operator $\mathrm{K}$ is obtained from the Dirac operator $K$ of [12] by a gauge transformation. Both operators are represented by weighted infinite adjacency matrices indexed by vertices of $G$. For $K$ the edges are unoriented and weighted by the critical weight function times a complex number of modulus 1. For K, edges are oriented with a clockwise odd orientation (see below), and are weighted by the critical weight function. Both weight functions yield the same Boltzmann measure on finite simply connected sub-graphs of $G$, but, and this is the reason why the real Dirac operator $\mathrm{K}$ is introduced, these weights do not yield the same probability distribution on toroidal subgraphs. Note that the real Dirac operator was already used in [3], see also [13].

\subsubsection{Definition}

Following Kasteleyn [8], let us define clockwise odd orientations on edges of $G$. An elementary cycle $\mathcal{C}$ of $G$ is said to be clockwise odd if, when traveling cw (clockwise) around the edges of $\mathcal{C}$, the number of co-oriented edges is odd. Note that since $G$ is bipartite, the number of contra-oriented edges is also odd. Then, an orientation of the edges of $G$ is defined to be clockwise odd if all elementary cycles are clockwise odd. In [8], Kasteleyn shows that, for planar simply connected graphs, such an orientation always exists.

Consider a clockwise odd orientation of the edges of $G$. Define $\mathrm{K}$ to be the infinite adjacency matrix of the graph $G$, weighted by the critical weight function $\nu$. That is, 
if $v_{1}$ and $v_{2}$ are not adjacent, $\mathrm{K}\left(v_{1}, v_{2}\right)=0$. If $w \in W$ and $b \in B$ are adjacent vertices, then $\mathrm{K}(w, b)=-\mathrm{K}(b, w)=(-1)^{\mathbb{I}_{(w, b)}} \nu(w b)$, where $\mathbb{I}_{(w, b)}=0$ if the edge $w b$ is oriented from $w$ to $b$, and 1 if it is oriented from $b$ to $w$. The infinite matrix $\mathrm{K}$ defines the real Dirac operator $\mathrm{K}: \mathbb{C}^{V(G)} \rightarrow \mathbb{C}^{V(G)}$, by

$$
(\mathrm{K} f)(v)=\sum_{u \in G} \mathrm{~K}(v, u) f(u)
$$

The matrix $\mathrm{K}$ is also called a Kasteleyn matrix for the underlying dimer model.

\subsubsection{Inverse real Dirac operator}

Similarly to the definition of the inverse Dirac operator $K^{-1}$ [12], the inverse real Dirac operator $\mathrm{K}^{-1}$ is defined to be the unique operator satisfying:

1. $\mathrm{KK}^{-1}=\mathrm{Id}$,

2. $\mathrm{K}^{-1}(b, w) \rightarrow 0$, when $|b-w| \rightarrow \infty$.

The rational functions $\mathrm{f}_{w x}(z)$ are the analog of the rational functions $f_{w v}(z)$ of [12], and are defined in the following way. Let $w \in W$, and let $x \in B$ (resp. $x \in W$ ); consider the edge-path $w=w_{1}, b_{1}, \ldots, w_{k}, b_{k}=x$ (resp. $\left.w=w_{1}, b_{1}, \ldots, w_{k}, b_{k}, w_{k+1}=x\right)$ of $G^{*}$ from $w$ to $x$. Let $R\left(w_{j} b_{j}\right)$ be the rhombus associated to the edge $w_{j} b_{j}$, and denote by $w_{j}, x_{j}, b_{j}, y_{j}$ its vertices in cclw (counterclockwise) order; $e^{i \alpha_{j}}$ is the complex vector $y_{j}-w_{j}$, and $e^{i \beta_{j}}$ is the complex vector $x_{j}-w_{j}$. In a similar way, denote by $w_{j+1}, x_{j}^{\prime}, b_{j}, y_{j}^{\prime}$ the vertices of the rhombus $R\left(w_{j+1} b_{j}\right)$ in cclw order, then $e^{i \alpha_{j}^{\prime}}$ is the complex vector $y_{j}^{\prime}-w_{j+1}$, and $e^{i \beta_{j}^{\prime}}$ is the complex vector $x_{j}^{\prime}-w_{j+1}$. The function $\mathrm{f}_{w x}(z)$ is defined inductively along the path,

$$
\begin{aligned}
& \mathrm{f}_{w w}(z)=1 \\
& \mathrm{f}_{w b_{j}}(z)=\mathrm{f}_{w w_{j}}(z) \frac{(-1)^{\mathbb{I}_{\left(w_{j}, b_{j}\right)}} e^{i \frac{\alpha_{j}+\beta_{j}}{2}}}{\left(z-e^{i \alpha_{j}}\right)\left(z-e^{i \beta_{j}}\right)}, \\
& \mathrm{f}_{w w_{j+1}}(z)=\mathrm{f}_{w b_{j}}(z)(-1)^{\mathbb{I}_{\left(w_{j+1}, b_{j}\right)}} e^{-i \frac{\alpha_{j}^{\prime}+\beta_{j}^{\prime}}{2}}\left(z-e^{i \alpha_{j}^{\prime}}\right)\left(z-e^{i \beta_{j}^{\prime}}\right) .
\end{aligned}
$$

By Lemma 9 of [3], the function $\mathrm{f}_{w x}$ is well defined. Moreover, Lemma 10 of [3] gives an explicit expression for $\mathrm{K}^{-1}$ (it is the analog of Theorem 4.2 of [12]):

Lemma 2 [3] The inverse real Dirac operator is given by

$$
\mathrm{K}^{-1}(b, w)=\frac{1}{4 \pi^{2} i} \int_{C} \mathbf{f}_{w b}(z) \log z d z,
$$

where $C$ is a closed contour surrounding cclw the part of the circle $\left\{e^{i \theta} \mid \theta \in\left[\theta_{0}-\pi+\right.\right.$ $\left.\left.\Delta, \theta_{0}+\pi-\Delta\right]\right\}$, which contains all the poles of $\mathrm{f}_{w b}$, and with the origin in its exterior. 


\subsection{Partition function of the torus}

In this section, we give the explicit formula of Kasteleyn [8] (in the $\mathbb{Z}^{2}$ case), and Tesler [21] (in the general periodic case) for the toroidal partition function $Z\left(G_{n}, \nu\right)$. Note that this result was already described in [3], we nevertheless choose to repeat it here since its understanding is important for the proof of Theorem 1.

Let us first orient the edges of $G$. Consider the graph $G_{1}$, it is a bipartite graph on the torus. Fix a reference matching $M_{0}$ of $G_{1}$. For every other perfect matching $M$ of $G_{1}$, consider the superposition $M \cup M_{0}$ of $M$ and $M_{0}$, then $M \cup M_{0}$ consists of doubled edges and cycles. Let us define four parity classes for perfect matchings $M$ of $G_{1}$ : (e,e) consists of perfect matchings $M$, for which cycles of $M \cup M_{0}$ circle the torus an even number of times horizontally and vertically; $(\mathrm{e}, \mathrm{o})$ consists of perfect matchings $M$, for which cycles of $M \cup M_{0}$ circle the torus and even number of times horizontally, and an odd number of times vertically; $(\mathrm{o}, \mathrm{e})$ and $(\mathrm{o}, \mathrm{o})$ are defined in a similar way. By Tesler [21], one can construct an orientation of the edges of $G_{1}$, so that the corresponding adjacency matrix $\mathrm{K}_{1}^{1}$ has the following property: perfect matchings which belong to the same parity class have the same sign in the expansion of the determinant; moreover of the four parity classes, three have the same sign and one the opposite sign. By an appropriate choice of sign, we can make the (e,e) class have the plus sign in $\operatorname{det} \mathrm{K}_{1}^{1}$, and the other three have minus sign. Consider a horizontal and a vertical cycle of the dual graph $G_{1}^{*}$ of $G_{1}$. Then define $\mathrm{K}_{2}^{1}$ (resp. $\mathrm{K}_{3}^{1}$ ) to be the matrix $\mathrm{K}_{1}^{1}$ where the sign of the coefficients corresponding to edges crossing the horizontal (resp. vertical) cycle is reversed; and define $\mathrm{K}_{4}^{1}$ to be the matrix $\mathrm{K}_{1}^{1}$ where the sign of the coefficients corresponding to the edges crossing both cycles are reversed. By Kasteleyn [8] (in the $\mathbb{Z}^{2}$ case), and Tesler [21] (in the general case), we have the following,

$$
Z\left(G_{1}, \nu\right)=\frac{1}{2}\left(-\operatorname{det} \mathrm{K}_{1}^{1}+\operatorname{det} \mathrm{K}_{2}^{1}+\operatorname{det} \mathrm{K}_{3}^{1}+\operatorname{det} \mathrm{K}_{4}^{1}\right) .
$$

The orientation of the edges of $G_{1}$ defines a periodic orientation of the graph $G$. For every $n$, consider the graph $G_{n}$, and the four matrices $\mathrm{K}_{1}^{n}, \mathrm{~K}_{2}^{n}, \mathrm{~K}_{3}^{n}, \mathrm{~K}_{4}^{n}$ defined as above. These matrices are called the Kasteleyn matrices of the graph $G_{n}$.

Theorem $3[8,21]$

$$
Z\left(G_{n}, \nu\right)=\frac{1}{2}\left(-\operatorname{det} \mathrm{K}_{1}^{n}+\operatorname{det} \mathrm{K}_{2}^{n}+\operatorname{det} \mathrm{K}_{3}^{n}+\operatorname{det} \mathrm{K}_{4}^{n}\right)
$$

The orientation defined on the edges of the graph $G$ is a clockwise odd orientation. Let K be the real Dirac operator indexed by the vertices of $G$, corresponding to this clockwise odd orientation. Note that except for edges crossing the horizontal and the vertical cycle, the coefficients of the Kasteleyn matrices $\mathrm{K}_{\ell}^{n}$ and of the real Dirac operator agree on edges they have in common. 


\subsection{Proof}

Consider an orientation of the edges of $G$ defined as in Section 2.2, and let $\mathrm{K}$ be the real Dirac operator indexed by vertices of $G$, corresponding to this orientation. Let $\mathrm{K}_{1}^{n}, \mathrm{~K}_{2}^{n}, \mathrm{~K}_{3}^{n}, \mathrm{~K}_{4}^{n}$ be the Kasteleyn matrices of the graph $G_{n}$. The following computations are inspired form [12]. By linear algebra, for every $\ell=1, \ldots, 4$, and for every edge $w_{i} b_{i}$ of $G_{n}$, we have

$$
\frac{\partial \operatorname{det} \mathbf{K}_{\ell}^{n}}{\partial\left(\mathbf{K}_{\ell}^{n}\left(w_{i}, b_{i}\right)\right)}=\left(\operatorname{det} \mathbf{K}_{\ell}^{n}\right)\left(\left(\mathbf{K}_{\ell}^{n}\right)^{-1}\left(b_{i}, w_{i}\right)\right) .
$$

Denote by $e_{1}=w_{1} b_{1}, \ldots, e_{m}=w_{m} b_{m}$ the edges of $G_{1}$, and let $\theta_{1}, \ldots, \theta_{m}$ be the corresponding rhombus angles. Since the graph $G_{n}$ is invariant under $\Lambda$-translates, we know that for every $\Lambda$-translate $w_{i}^{t} b_{i}^{t}$ of the edge $w_{i} b_{i}$, the coefficient $\mathrm{K}_{\ell}^{n}\left(w_{i}^{t}, b_{i}^{t}\right)=$ $\pm \mathbf{K}_{\ell}^{n}\left(w_{i}, b_{i}\right)$. The minus sign only occurs when $\ell=2,3,4$, (recall that in the definition of $\mathrm{K}_{2}^{n}, \ldots, \mathrm{K}_{4}^{n}$, the sign of the entries which cross the horizontal and/or the vertical cycle of $G_{n}^{*}$ is reversed), but then $\left(\mathrm{K}_{\ell}^{n}\right)^{-1}\left(b_{i}^{t}, w_{i}^{t}\right)= \pm\left(\mathrm{K}_{\ell}^{n}\right)^{-1}\left(b_{i}, w_{i}\right)$. Hence, for every $\ell=1, \ldots, 4$, and for every $i=1, \ldots, m$,

$$
\begin{aligned}
\frac{\partial \operatorname{det} \mathrm{K}_{\ell}^{n}}{\partial \theta_{i}} & =\sum_{w_{i}^{t} b_{i}^{t} \text { translates of } w_{i} b_{i}} \frac{\partial \operatorname{det} \mathrm{K}_{\ell}^{n}}{\partial\left(\mathrm{K}_{\ell}^{n}\left(w_{i}^{t}, b_{i}^{t}\right)\right)} \frac{\partial\left(\mathrm{K}_{\ell}^{n}\left(w_{i}^{t}, b_{i}^{t}\right)\right)}{\partial \theta_{i}} \\
& =n^{2}\left(\operatorname{det} \mathrm{K}_{\ell}^{n}\right)\left(\left(\mathrm{K}_{\ell}^{n}\right)^{-1}\left(b_{i}, w_{i}\right)\right) \frac{\partial \mathrm{K}_{\ell}^{n}\left(w_{i}, b_{i}\right)}{\partial \theta_{i}}
\end{aligned}
$$

where the edges $e_{1}, \ldots, e_{m}$ do not cross the horizontal and the vertical path of the dual graph $G_{n}^{*}$ of $G_{n}$. Define the function $\varphi_{n}\left(\theta_{1}, \ldots, \theta_{m}\right)$ by

$$
\varphi_{n}\left(\theta_{1}, \ldots, \theta_{m}\right)=\frac{1}{n^{2}} \log Z\left(G_{n}, \nu\right) .
$$

By Theorem 3, we have $Z\left(G_{n}, \nu\right)=\frac{1}{2}\left(-\operatorname{det} \mathrm{K}_{1}^{n}+\operatorname{det} \mathrm{K}_{2}^{n}+\operatorname{det} \mathrm{K}_{3}^{n}+\operatorname{det} \mathrm{K}_{4}^{n}\right)$. Moreover, for every $\ell=1, \ldots, 4, \mathrm{~K}_{\ell}^{n}\left(w_{i}, b_{i}\right)=\mathrm{K}\left(w_{i}, b_{i}\right)$, so that using (5), we obtain for every $i=1, \ldots, m$,

$$
\frac{\partial \varphi_{n}}{\partial \theta_{i}}\left(\theta_{1}, \ldots, \theta_{m}\right)=\left(\frac{\partial \mathrm{K}\left(w_{i}, b_{i}\right)}{\partial \theta_{i}}\right)\left(\frac{-\operatorname{det} \mathrm{K}_{1}^{n}}{2 Z\left(G_{n}, \nu\right)}\left(\mathrm{K}_{1}^{n}\right)^{-1}\left(b_{i}, w_{i}\right)+\sum_{\ell=2}^{4} \frac{\operatorname{det} \mathrm{K}_{\ell}^{n}}{2 Z\left(G_{n}, \nu\right)}\left(\mathrm{K}_{\ell}^{n}\right)^{-1}\left(b_{i}, w_{i}\right)\right) .
$$

The next part of the argument can be found in [9]. The second bracket of equation (6) is a weighted average of the four quantities $\left(\mathrm{K}_{\ell}^{n}\right)^{-1}\left(b_{i}, w_{i}\right)$, with weights $\pm \frac{1}{2} \operatorname{det} \mathrm{K}_{\ell}^{n} / Z\left(G_{n}, \nu\right)$. These weights are all in the interval $(-1 / 2,1 / 2)$ since for every $\ell=1, \ldots, 4,2 Z\left(G_{n}, \nu\right)>$ $\left|\operatorname{det} \mathrm{K}_{\ell}^{n}\right|$. Indeed, $Z\left(G_{n}, \nu\right)$ counts the weighted sum of dimer configurations of $G_{n}$, whereas $\left|\operatorname{det} \mathrm{K}_{\ell}^{n}\right|$ counts some configurations with negative sign. Since the weights sum to 1 , the weighted average converges to the same value as each $\left(\mathrm{K}_{\ell}^{n}\right)^{-1}\left(b_{i}, w_{i}\right)$.

By Proposition 2 of [3], for every $\ell=1, \ldots, 4,\left(\mathrm{~K}_{\ell}^{n}\right)^{-1}\left(b_{i}, w_{i}\right)$ converges to $\mathrm{K}^{-1}\left(b_{i}, w_{i}\right)$ on a subsequence $\left(n_{j}\right)$ of $n$ 's. Hence, for every $\varepsilon>0$, there exists $n_{0}$ such that for $n \geq n_{0}, n \in\left(n_{j}\right)$, equation (6) can be written as

$$
\frac{\partial \varphi_{n}}{\partial \theta_{i}}\left(\theta_{1}, \ldots, \theta_{m}\right)=\frac{\partial \mathrm{K}\left(w_{i}, b_{i}\right)}{\partial \theta_{i}} \mathrm{~K}^{-1}\left(b_{i}, w_{i}\right)+\varepsilon
$$


Let us compute the right hand side of (7), using the notations of Section 2.1.2. By definition, $\mathrm{K}\left(w_{i}, b_{i}\right)=(-1)^{\mathbb{I}\left(w_{i}, b_{i}\right)} 2 \sin \theta_{i}$, hence

$$
\frac{\partial \mathrm{K}\left(w_{i}, b_{i}\right)}{\partial \theta_{i}}=(-1)^{\mathbb{I}\left(w_{i}, b_{i}\right)} 2 \cos \theta_{i} .
$$

Moreover, by definition $\mathrm{f}_{w_{i} b_{i}}(z)=(-1)^{\mathbb{I}\left(w_{i}, b_{i}\right)} \frac{e^{i \frac{\left(\alpha_{i}+\beta_{i}\right)}{2}}}{\left(z-e^{\left.i \alpha_{i}\right)\left(z-e^{i \beta_{i}}\right)}\right.}$. So that using Lemma 2, and the Residue Theorem yields:

$$
\begin{aligned}
\mathrm{K}^{-1}\left(b_{i}, w_{i}\right) & =\frac{1}{4 \pi^{2} i}(-1)^{\mathbb{I}\left(w_{i}, b_{i}\right)} e^{i \frac{\left(\alpha_{i}+\beta_{i}\right)}{2}} \int_{C} \frac{\log z}{\left(z-e^{i \alpha_{i}}\right)\left(z-e^{i \beta_{i}}\right)} d z, \\
& =\frac{1}{2 \pi}(-1)^{\mathbb{I}\left(w_{i}, b_{i}\right)} e^{i \frac{\left(\alpha_{i}+\beta_{i}\right)}{2}}\left(\frac{i \alpha_{i}}{e^{i \alpha_{i}}-e^{i \beta_{i}}}+\frac{i \beta_{i}}{e^{i \beta_{i}-\alpha_{i}}}\right) \\
& =\frac{1}{2 \pi}(-1)^{\mathbb{I}\left(w_{i}, b_{i}\right)} \frac{\theta_{i}}{\sin \theta_{i}} .
\end{aligned}
$$

Combining equations (8) and (9), implies:

$$
\frac{\partial \varphi_{n}}{\partial \theta_{i}}\left(\theta_{1}, \ldots, \theta_{m}\right)=\frac{\theta_{i}}{\pi} \operatorname{cotan} \theta_{i}+\varepsilon
$$

By [12], there is a continuous way to deform the graph $G$ so that all rhombus angles tend to 0 or $\pi / 2$. The same transformation can be applied to $G_{n}$, for every $n$. Denote by $\theta_{i}^{0}$ the angle $\theta_{i}$ after such a deformation. Let $M$ be the number of angles $\theta_{1}^{0}, \ldots, \theta_{m}^{0}$ which are equal to $\pi / 2$. By the argument of [12], for $n \geq n_{0}, n \in\left(n_{j}\right)$, we obtain

$$
\varphi_{n}\left(\theta_{1}, \ldots, \theta_{m}\right)=\sum_{i=1}^{m}\left(\frac{\theta_{i}}{\pi} \log 2 \sin \theta_{i}+\frac{1}{\pi} L\left(\theta_{i}\right)\right)-\frac{M}{2} \log 2+\varphi_{n}\left(\theta_{1}^{0}, \ldots, \theta_{m}^{0}\right)+\varepsilon .
$$

Let us compute $\varphi_{n}\left(\theta_{1}^{0}, \ldots, \theta_{m}^{0}\right)$. Suppose the above deformation is applied to the graph $G$, then edges corresponding to rhombus angles 0 have weight 0 , and those corresponding to rhombus angles $\pi / 2$ have weight 2 . Removing the 0 weight edges, the deformed graph consists of independent copies of $\mathbb{Z}$. Applying the deformation to $G_{1}$, we obtain graphs $\mathbb{Z} / m_{1} \mathbb{Z}, \ldots, \mathbb{Z} / m_{p} \mathbb{Z}$, for even $m_{1}, \ldots, m_{p}$, where each $\mathbb{Z} / m_{j} \mathbb{Z}$ consists of weight 2 edges. We can compute,

$$
Z\left(G_{n}, \nu\right)=\left(2^{\frac{n m_{1}}{2}} 2\right)^{n} \ldots\left(2^{\frac{n m_{p}}{2}} 2\right)^{n}=2^{n^{2} \frac{M}{2}} 2^{n p} .
$$

Hence, for every $\varepsilon>0$, there exists $n_{1}$, such that for every $n \geq n_{1}$, we have

$$
\varphi_{n}\left(\theta_{1}^{0}, \ldots, \theta_{m}^{0}\right)=\frac{M}{2} \log 2+\varepsilon .
$$

Combining equations (10) and (11) yields, for every $n \geq \max \left\{n_{0}, n_{1}\right\}, n \in\left(n_{j}\right)$,

$$
\varphi_{n}\left(\theta_{1}, \ldots, \theta_{m}\right)=\sum_{i=1}^{m}\left(\frac{\theta_{i}}{\pi} \log 2 \sin \theta_{i}+\frac{1}{\pi} L\left(\theta_{i}\right)\right)+2 \varepsilon .
$$


This implies,

$$
\lim _{n \rightarrow \infty}^{\prime} \varphi_{n}\left(\theta_{1}, \ldots, \theta_{m}\right)=\sum_{i=1}^{m}\left(\frac{\theta_{i}}{\pi} \log 2 \sin \theta_{i}+\frac{1}{\pi} L\left(\theta_{i}\right)\right) .
$$

where the limit is taken on the subsequence $\left(n_{j}\right)$. Using Theorem 3.5 of [14], we deduce that $\varphi_{n}\left(\theta_{1}, \ldots, \theta_{m}\right)$ converges (as $n \rightarrow \infty$ ) to the same limit as the above subsequence.

\section{References}

[1] R. J. Baxter, Exactly solved models in statistical mechanics. Academic Press, London (1982).

[2] H. Cohn, R. Kenyon, J. Propp, A variational principle for domino tilings. J. Amer. Math. Soc. 14 (2001), no 2, 297-346.

[3] B. de Tilière, Quadri-tilings of the plane. Probab. Theory Relat. Fields http://dx.doi.org/10.1007/s00440-006-0002-9

[4] B. de Tilière, Dimères sur les graphes isoradiaux \& modèle d'interfaces aléatoires en dimension $2+2$. PhD Thesis. Université Paris XI, Orsay (2004).

[5] R. J. Duffin, Potential theory on a rhombic lattice. J. Combinatorial Theory 5 (1968), 258-272.

[6] N. Elkies, G. Kuperberg, M. Larsen, and J. Propp, Alternating-sign matrices and domino tilings. J. Algebraic Combin. 1 (1992), no 2, 111-132.

[7] P.W. Kasteleyn, The statistics of dimers on a lattice, I. The number of dimer arrangements on a quadratic lattice. Physica 27 (1961), 1209-1225.

[8] P.W. Kasteleyn, Graph theory and crystal physics. Graph Theory and Theoretical Physics, Academic Press, London, (1967), 43-110.

[9] R. Kenyon, Local statistics of lattice dimers. Ann. Inst. H. Poincaré, Probab. Statist. 33 (1997), no 5, 591-618.

[10] R. Kenyon, An introduction to the dimer model. Lecture notes for a short course at the ICTP, (May 2002).

[11] R. Kenyon, The planar dimer model with boundary: a survey. CRM Monogr. Ser. 13, Amer. Math. Soc., Providence, (2000), 307-328.

[12] R. Kenyon, The Laplacian and Dirac operators on critical planar graphs. Invent. Math. 150 (2002), no 2, 409-439. 
[13] R. Kenyon, A. Okounkov, Planar dimers and Harnack curves. math-ph/0311005. To appear, Duke Math. J.

[14] R. Kenyon, A. Okounkov, S. Sheffield, Dimers and amoebas. math-ph/0311005. To appear, Ann. Math.

[15] G. Kuperberg, An exploration of the permanent-determinant method. Electron. J. Combin. 5 (1998), Research Paper 46, 34 pp. (electronic).

[16] C. Mercat, Discrete Riemann surfaces and the Ising model. Comm. Math. Phys. 218 (2001), no. 1, 177-216.

[17] C. Mercat, Exponentials form a basis of discrete holomorphic functions on a compact. Bull. Soc. Math. France 132 (2004), no. 2, 305-326.

[18] C. Mercat, Discrete period matrices and related topics. math-ph/0111043.

[19] S. Sheffield, PhD Thesis. Stanford University (2003).

[20] H. N. V. Temperley, M. E. Fisher, Dimer problem in statistical mechanics - An exact result. Phil. Mag. 6 (1961), 1061-1063.

[21] G. Tesler, Matchings in graphs on non-orientable surfaces. J. Combin. Theory Ser. B 78 (2000), no. 2, 198-231. 